\title{
Induction of Phenylalanine and Tyrosine Oxidation in the Intoxicated Larva of the Silkworm, Bombyx mori L (Lepidoptera : Bombycidae), with MTMC ( $m$-Tolyl- $N$-methylcarbamate)
}

\author{
Hirosi Sugiyama, Hiroshi Moriyama and Hajime Shigematsu
}

The Sericulturai Experiment Station, Yatabe, Tsukuba, Ibaraki 305, Japan

(Received June 24, 1981)

\begin{abstract}
Labeled phenylalanine or tyrosine administered to normal 5th instar larvae of the silkworm, Bombyx mori L (Lepidoptera : Bombycidae), was metabolized by sclerotization, that is, from phenylalanine to tyrosine, Dopa and Dopamine. Whereas the MTMC ( $m$-tolyl$\mathrm{N}$-methylcarbamate) treatment altered the metabolism of aromatic amino acids in the silkworm, the interconversion between phenylalanine and tyrosine was almost completely blocked and $80 \%$ of the phenylalanine was converted into PLA (phenyllactic acid). The MTMG treatment induced formation of a significant amount of HPLA ( $p$-hydroxyphenyllactic acid) from tyrosine, but it did not change the conversion of tyrosine into Dopa. Consequently, it was shown that MTMG treatment induced the deamination of phenylalanine and tyrosine, while it made normal and steady progress in sclerotization.
\end{abstract}

\section{INTRODUCTION}

We previously noticed the accumulation of HPLA( $p$-hydroxyphenyllactic acid) in silkworm larvae intoxicated with MTMC (Sugryama et al., 1981). Two direct precursors are supposed to be HPPA( $p$-hydroxyphenylpyruvic acid) resulting from the deamination of tyrosine, and PLA(phenyllactic acid) from phenylalanine by deamination and oxidation. KARLSON and SEKERIS (1962) proposed two ways of tyrosine metabolism in the blow-fly, Calliphora erythrocephala, by oxidative degradation and sclerotization specified to the early $3 \mathrm{rd}$ instar and the late $3 \mathrm{rd}$ instar, respectively. HPPA and HPLA are members of the former pathway. This stirred our interest in the manner of formation of HPLA and in the main route of tyrosine and phenylalanine metabolism in silkworm larvae intoxicated with MTMG. Some distinct results were obtained in the reported experiments.

\section{MATERIALS AND METHODS}

Silkworm. Six-day-old larvae of the 5th instar of the silkworm, Bombyx mori, Nichi $124 \times$ Shi 124 , were used in these experiments.

Standard compounds for TLC. L-phenylalanine, L-tyrosine, Dopa and Dopamine were purchased from Wako Pure Chemical Industries, Ltd., Osaka, Japan. PPA (phenylpyruvic acid), PLA and HPPA were purchased from Sigma Chemical Co., 
Missouri, U.S.A.. HPLA was prepared as previously reported (Sugryama et al., 1981).

Application of $M T M C$ to larvae. MTMG ( $m$-tolyl- $N$-methylcarbamate) was obtained from Hokko Chemical Industry Co. Ltd., Tokyo, Japan. Ten $\mu$ g of MTMG was dissolved in acetone and was applied topically one time to each larva per body weight, $\mathrm{g}$.

Administration of labeled phenylalanine and tyrosine. $\mathrm{L}-\left(\mathrm{U}-{ }^{14} \mathrm{C}\right)$ phenylalanine $(513$ $\mathrm{mCi} / \mathrm{m}$ mole $)$ and $\mathrm{L}_{-}\left(\mathrm{U}_{-}{ }^{14} \mathrm{C}\right)$ tyrosine $(531 \mathrm{mCi} / \mathrm{m}$ mole) were purchased from The Radiochemical Centre, Amersham, England. After MTMG treatment each larva was administered with $1 \mu \mathrm{Ci}$ of phenylalanine or $0.5 \mu \mathrm{Ci}$ of tyrosine.

Collection of the haemolymph. At various hours after administration of labeled amino acids, the haemolymph of either the intoxicated or the normal larvae was collected in separate small sample vials, chilled in acetone-dry ice from the thoracic legs, and then was kept at $-20^{\circ} \mathrm{C}$ until used.

$T L C$ analysis for key metabolites of phenylalanine and tyrosine. Phenylalanine and its metabolites were isolated by means of TLC with a solvent system of $n$-propylalcohol$28 \% \mathrm{NH}_{4} \mathrm{OH}(67: 33, \mathrm{v} / \mathrm{v})$. Rf values afforded were as follows: phenylalanine 0.63 , tyrosine 0.48, Dopa 0-0.4 (tailing), PLA \& PPA 0.68-0.72, and HPLA 0.91. Tyrosine and its metabolites were isolated with $n$-butylalcohol-acetic acid- $\mathrm{H}_{2} \mathrm{O}(6: 2: 2$, $\mathrm{v} / \mathrm{v}$ ) system. Each $\mathrm{Rf}$ was obtained as follows: tyrosine 0.57, Dopa 0.48, Dopamine 0.61 , HPPA 0.84 and HPLA 0.94. TLG plates attached with condensation zone (Merck, silica gel 60) were used. After development, the plates were exposed to iodine vapour to visualize the spots. The spots corresponding to the derived compounds from labeled amino acids in $5 \%$ PCA soluble fraction of the haemolymph were scraped off the plates after co-chromatography with these standard substances and their radioactivity was measured.

\section{RESULTS}

\section{Metabolism of phenylalanine}

We measured the radioactivity appearing in the key metabolites of either oxidative degradation or sclerotization every hour after the administration of the labeled phenylalanine. PLA and HPLA were assayed as the key metabolites of oxidative degradation, and Dopa of sclerotization. Results are presented in Fig. 1. The hydroxylation rate of phenylalanine to tyrosine differed greatly between the control and intoxicated larvae. In the control larvae phenylalanine was rapidly transformed into tyrosine and was hardly detectable, whereas, in the intoxicated larvae, less than a quarter of the phenylalanine could be hydroxylated to tyrosine (Fig. 1 A,B). The radioactivity of phenylalanine which remaining unchanged was completely recovered in a fraction containing PLA and PPA in the intoxicated larvae. In the control, PLA plus PPA fractions could hardly be labeled (Fig. l C), but the radioactivity of Dopa was highly detectable, compared with the intoxicated larvae (Fig. 1 D). A late labeling was seen in HPLA of the intoxicated larvae (Fig. $1 \mathrm{E}$ ), but its radioactivity was only $3 \%$ of PLA plus PPA.

Calculation of transfer of the radioactivity among the metabolites derived in the intoxicated larvae showed that of phenylalanine taken up $80 \%$ was transferred into PLA plus PPA and the remaining 20\% was converted into tyrosine; $30 \%$ of this 

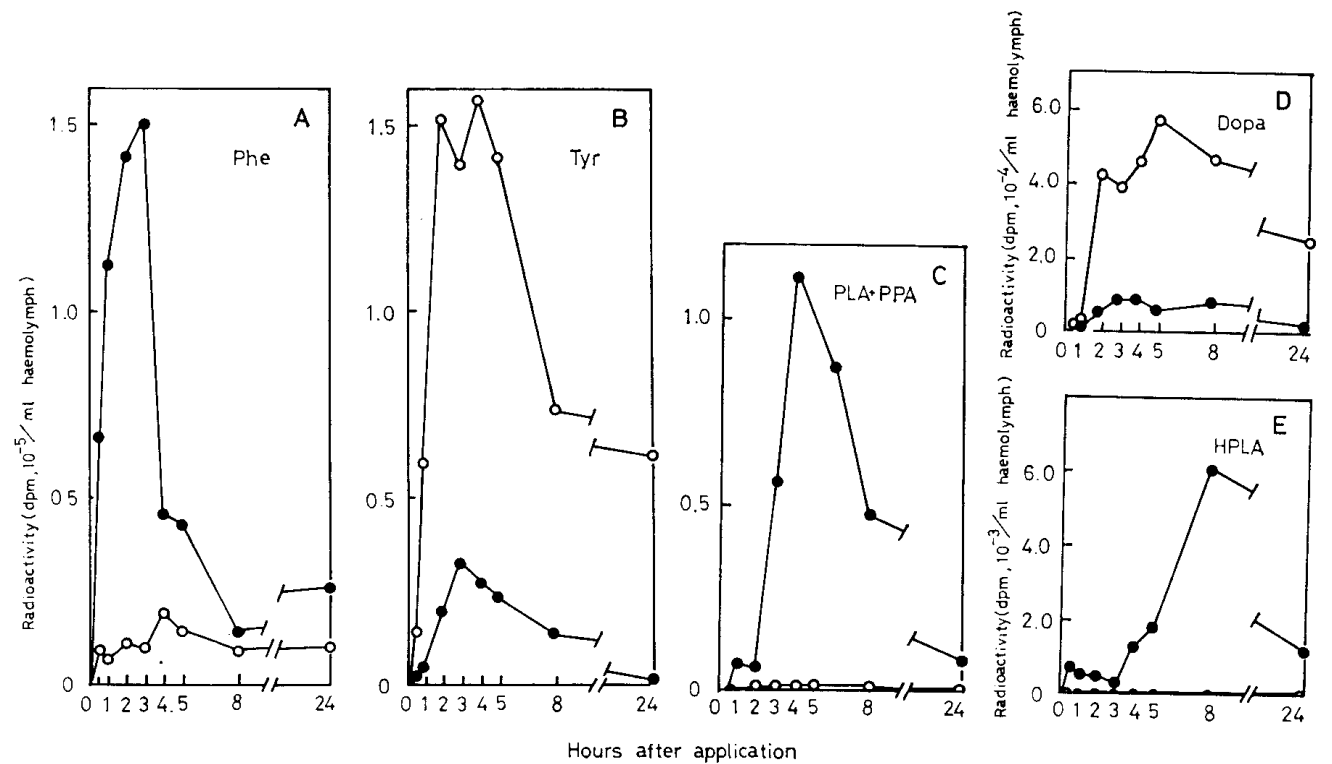

Fig. 1. Change in the radioactivity found in phenylalanine metabolites of the haemolymph from intoxicated larvae. - $\mathrm{O}-$ Control

latter served to produce Dopa and Dopamine. The ability to produce Dopa and Dopamine from phenylalanine was about equal in the control and the intoxicated larvae.

Table 1 presents the ratio of radioactivity found in each key metabolite derived

Table 1. Change in the ratio of phenylalanine metabolites in the haemolymph during the MTMC exposure

\begin{tabular}{ccccccc}
$\begin{array}{c}\text { Metabolites } \\
\text { hours after } \\
\text { application }\end{array}$ & Phe & Try & Dopa & PLA +PPA & HPLA & $\begin{array}{c}\text { Radioactivity of } \\
\text { supernatant } \\
\left(10^{-5} / \mathrm{m} l\right)\end{array}$ \\
\hline $\begin{array}{c}\text { Treated } \\
0.5\end{array}$ & $94.2 \%$ & $3.0 \%$ & $0.7 \%$ & $0.7 \%$ & $1.1 \%$ & $0.71 \mathrm{dpm}$ \\
1 & 89.5 & 3.7 & 0.1 & 5.1 & 0.4 & 1.38 \\
2 & 63.1 & 9.1 & 2.2 & 2.6 & 0.2 & 2.26 \\
3 & 61.8 & 13.7 & 3.4 & 23.0 & 0.1 & 2.44 \\
4 & 23.8 & 14.7 & 4.3 & 57.1 & 0.7 & 1.95 \\
5 & 27.0 & 14.9 & 3.7 & 53.5 & 1.2 & 1.63 \\
8 & 15.7 & 15.9 & 8.9 & 52.8 & 6.8 & 0.91 \\
24 & 64.6 & 4.8 & 5.0 & 22.7 & 2.7 & 0.41 \\
Control & & & & & & \\
0.5 & 35.8 & 59.2 & 3.3 & 1.6 & n.d. & 0.25 \\
1 & 8.7 & 87.9 & 3.3 & 0.6 & n.d. & 0.68 \\
2 & 5.4 & 73.6 & 20.5 & 0.3 & n.d. & 2.06 \\
3 & 5.6 & 73.4 & 20.6 & 0.4 & n.d. & 1.92 \\
4 & 8.5 & 70.5 & 20.9 & 0.1 & n.d. & 2.25 \\
5 & 6.8 & 66.5 & 26.7 & n.d. & n.d. & 2.14 \\
8 & 7.1 & 57.1 & 35.2 & n.d. & n.d. & 1.31 \\
24 & 10.7 & 64.4 & 24.8 & n.d. & n.d. & 0.97 \\
& & & & &
\end{tabular}


from phenylalanine to the whole activity of the haemolymph supernatant. Several indices which indicate the interrelationship between metabolites can be inferred from this table. The ratio of Dopa to tyrosine was almost steady for a period of time after a $2 \mathrm{hr}$ lapse of ${ }^{14} \mathrm{C}$-phenylalanine exposure in the control and between a $3 \mathrm{hr}$ and 5 hr lapse in the intoxicated larvae. During this period, the ratio of tyrosine to Dopa was constant at about $70: 20$. Peculiar phenomena appearing in the intoxicated larvae were as follows: more than half of the activity was held by PLA plus PPA between a $4 \mathrm{hr}$ and $8 \mathrm{hr}$ lapse, and HPLA was labeled late at a significant ratio.

\section{Metabolism of tyrosine}

After the administration of labeled tyrosine to the larvae, transfer of the radio-
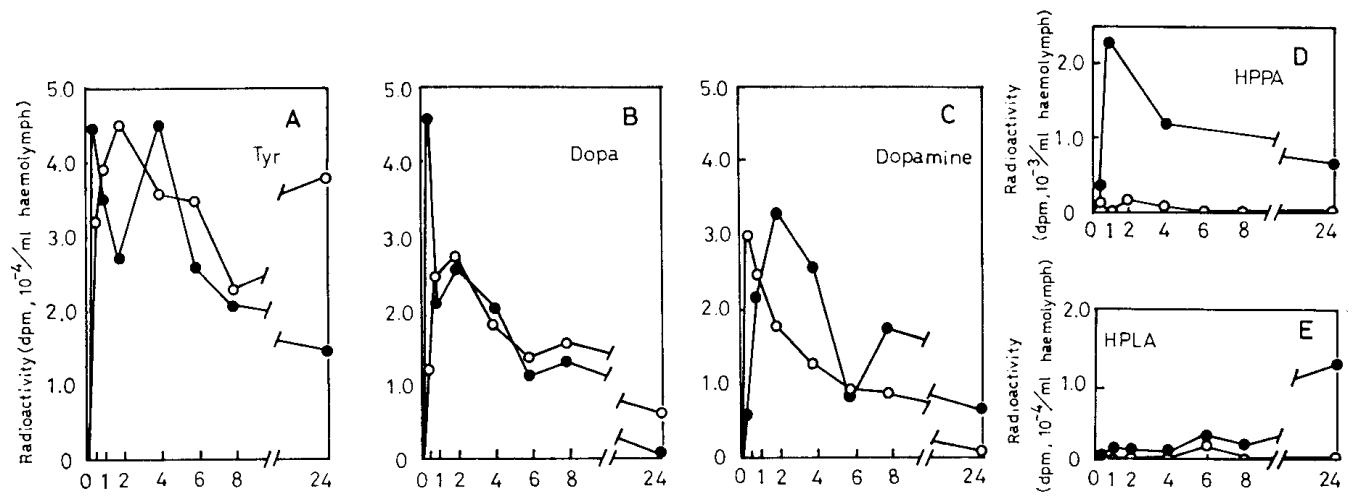

Hours after application

Fig. 2. Change in the radioactivity found in tyrosine metabolites of the haemolymph from intoxicated larvae. - $\mathrm{O}-$ Control

- Intoxicated with MTMG

Table 2. Change in the ratio of tyrosine metabolites in the haemolymph during the MTMG exposure

\begin{tabular}{ccccccc}
$\begin{array}{c}\text { Metabolites } \\
\text { hours after } \\
\text { application }\end{array}$ & Tyr & Dopa & Dopamine & HPPA & HPLA & $\begin{array}{c}\text { Radioactivity of } \\
\text { supernatant } \\
\left(10^{-5} / \mathrm{ml}\right)\end{array}$ \\
\hline $\begin{array}{c}\text { Treated } \\
0.5\end{array}$ & $46.5 \%$ & $47.1 \%$ & 6.0 & $0.4 \%$ & n.d.\% & $0.96 \mathrm{dpm}$ \\
1 & 43.7 & 25.8 & 26.6 & 2.8 & 1.1 & 0.81 \\
2 & 31.6 & 29.5 & 37.9 & - & 1.0 & 0.87 \\
3 & 48.9 & 21.8 & 27.4 & 1.2 & 0.7 & 0.93 \\
6 & 56.0 & 20.6 & 16.6 & - & 6.8 & 0.46 \\
8 & 39.2 & 24.3 & 33.1 & - & 3.4 & 0.53 \\
24 & 37.8 & 13.8 & 16.0 & 1.7 & 30.7 & 0.40 \\
Control & 43.9 & 16.3 & 39.6 & 0.02 & n.d. & 0.74 \\
0.5 & 44.2 & 27.8 & 28.0 & - & n.d. & 0.88 \\
1 & 50.1 & 29.9 & 19.7 & 0.03 & n.d. & 0.90 \\
2 & 53.8 & 27.1 & 18.8 & 0.02 & n.d. & 0.67 \\
4 & 59.3 & 23.2 & 14.5 & - & 3.0 & 0.59 \\
6 & 48.8 & 33.5 & 17.6 & - & n.d. & 0.47 \\
8 & 100.0 & n.d. & n.d. & - & n.d. & 0.38 \\
24 & & & & & & \\
\hline
\end{tabular}


activity from tyrosine to Dopa, Dopamine, HPPA and HPLA was traced. Results are shown in Fig. 2. Labeled tyrosine was retained in the haemolymph a relatively long time and then was converted into Dopa and Dopamine (Fig. 2 A,B,C). There was neither acceleration nor retardation of this conversion by the MTMC treatment, compared with the control. The reaction of tyrosine metabolism proceeded normally in the ordinary way of sclerotization even in the intoxicated larvae. On the contrary, labeling of HPPA and HPLA was induced only by MTMC treatment, unobserved in the control (Fig. 2 D,E). Most of the radioactivity which had first appeared in HPPA was transferred to HPLA within several hours. Though labeling of HPLA was late, almost $20 \%$ of the radioactivity of tyrosine found initially was recovered in HPLA $24 \mathrm{hr}$ after application.

Table 2 shows the ratio of radioactivity found in the metabolites to total activity of haemolymph. Conversion of tyrosine into Dopa was likely to be steady between 1 and $6 \mathrm{hr}$ after administration with the tracer in each of control and intoxicated, since the ratio of tyrosine to Dopa was almost constant. The ratio of HPLA started to appear $6 \mathrm{hr}$ after administration, and went up as high as $30 \%$.

\section{DISGUSSION}

From these experiments with 6-day-old 5th instar control silkworm larvae, it is concluded that phenylalanine is rapidly converted into tyrosine by hydroxylation (Fukuda, 1956), being presumably non-specific, and then takes progress of sclerotization (Karlson and Sereris, 1962; Karlson, 1965). About 40\% of the phenylalanine was recovered as Dopa or Dopamine. On the other hand, in larvae intoxicated with MTMC, deamination of phenylalanine was converted into PLA and tyrosine into HPLA. Of the phenylalanine taken up, $80 \%$ was metabolized to PLA and about $30 \%$ of the tyrosine, which was formed by the minor conversion, became Dopamine.

Restrained hydroxylation activity caused by MTMG treatment and found in the conversion of phenylalanine into tyrosine, lead us to consider that the conversion of PPA into HPPA or of PLA into HPLA is not likely. HPLA was formed by successive function of oxidative deamination of tyrosine and reduction of HPPA, on the way of oxidative degradation (KARLSON and SEKERIS, 1962). Action of MTMG on the metabolism of phenolic amino acids was verified to be a block of hydroxylation of tyrosine and an induction of deamination of phenylalanine and tyrosine.

Significance of the accumulation of HPLA, which has carcinogenic action in mammals (Zharova et al., 1979), is unknown but seems to be one of the symptoms which are unfavorable to the animal. In fact, patients with phenylketonuria produce an abnormally large amount of L-phenyllactate, which would inhibit oxalate synthesis (Ghernoff et al., 1978). Other patients with hepatic diseases show symptoms accompanied by an increase of serum HPPA (Ruge and Отто, 1969). In the course of restoration of intoxicated larvae, accumulated HPLA and PLA act as pools for tyrosine and phenylalanine formation, respectively. In mammals, although not in insects, perfusion of tissues with phenylpyruvate leads to the appearance of tyrosine (WALSER et al., 1973).

Finally, the proposed pathway of phenylalanine and tyrosine metabolism functioning in intoxicated larvae is presented in Fig. 3. 


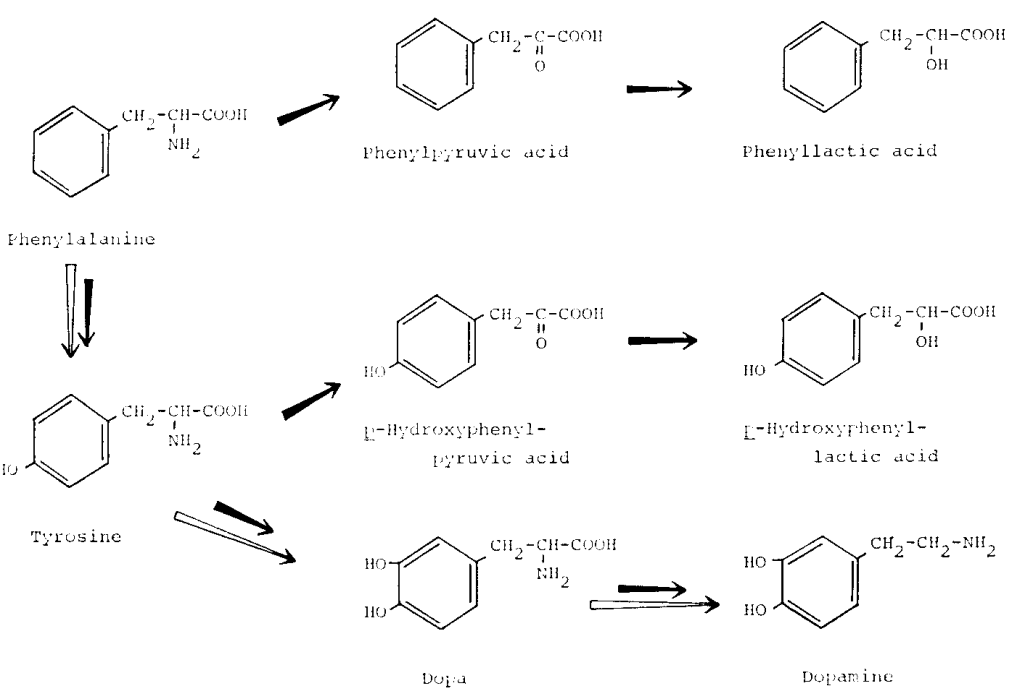

Fig. 3. Proposed pathway of phenylalanine and tyrosine metabolism in Bombyx. $\Rightarrow$ Control $\Rightarrow$ Intoxicated with MTMG

\section{ACKNOWLEDGMENTS}

We wish to express our thanks to Dr. M. Koвayashi of The Sericultural Experiment Station for reading this manuscript. This work was partly supported by the Environmental Agency, Japan.

\section{REFERENGES}

Cihernoff, H. N. and K. E. Richardson (1978) The effect of endogenous L-phenyllactate on oxalate, glycolate, and glyoxylate excretion by phenylketonuric subjects. Clin. Chim. Acta 83 : 1-6.

Fukuda, T. (1956) Conversion of phenylalanine into tyrosine in the silkworm larva (Bombyx mori). Nature 4505 : 429-430.

KarLson, P. (1965) Biochemical studies of ecdysone control of chromosomal activity. J. Cell. and Comp. Physiol. $66: 69-76$.

Karlson, P. and C. E. SeKeris (1962) N-Acetyl-dopamine as sclerotizing agent of the insect cuticle. Nature 4839 : 183-184.

Ruge, W. and P. Oтto (1969) Metabolism of phenolic compounds in hepatic diseases with encephalopathic effects. Verh. Deut. Ges. Inn. Med. $75: 329-332$.

Sugryama, H., H. Moriyama and H. Shigematsu (1981) Specific accumulation of $p$-hydroxyphenyllactic acid as a metabolite of tyrosine metabolism in the haemolymph of the larva of Bombyx mori $\mathrm{L}$. (Lepidoptera : Bombycidae) intoxicated with MTMG (m-tolyl- $N$-methylcarbamate). Appl. Ent. Zool. 16 : 472-476,

Walser, M., P. Lund and N. B. Ruderman (1973) Synthesis of essential amino acids from their $\alpha$-keto analogs by perfused rat liver and muscle. J. Clin. Invest. 52 : 2865-2877.

Zharova, E. I., T. I. Sergeeva, N. V. Makhalova, V. I. Romanenko, N. G. Chitiridi and M. O. RAlishenbakH (1979) Transplacental blastomogenic action of $p$-hydroxyphenyllactic acid. Eksp. Biol. Med. $87: 39-41$. 